\title{
AVALIAÇÃO DO MÉTODO DE PRODAN NA AMOSTRAGEM DE CERRADO SENSU STRICTO NO DISTRITO FEDERAL
}

\author{
Erica Karolina Barros de OLIVEIRA ${ }^{1}$ \\ Lidiomar Soares da COSTA ${ }^{2}$ \\ Ilvan Medeiros LUSTOSA JÚNIOR ${ }^{3}$ \\ Leonidas Soares MURTA JÚNIOR ${ }^{4}$ \\ Hugo Macedo BUCHMANN ${ }^{1}$ \\ Fabrícia Conceição Menez MOTA ${ }^{1}$ \\ Alba Valéria REZENDE ${ }^{1}$
}

- RESUMO: Este estudo objetivou avaliar e comparar os métodos de amostragem de Área Fixa e Prodan em Cerrado sensu stricto no Distrito Federal. Foram distribuídas aleatoriamente dez unidades amostrais na área de estudo, com dimensões de $20 \times 50$ m para o método de Área Fixa, no centro de cada uma dessas foram demarcados os pontos para amostragem pelo método de Prodan. Em cada unidade, as árvores foram identificadas e tiveram o diâmetro na base $(D b \geq 5$ $\mathrm{cm}$ ) e a altura total mensurados. Pelo método de Prodan, foram amostradas 39,1\% das espécies e $60,7 \%$ das famílias botânicas amostradas pelo método de Área Fixa. Espécies amostradas por Prodan foram, em maioria, aquelas com maiores valores de frequência, densidade e dominância relativas obtidas no método de Área Fixa. O número de árvores, área basal e volume por hectare não diferiram entre os dois métodos. $\mathrm{O}$ método de Prodan, embora amostre menor número de espécies e famílias botânicas, amostra as espécies mais representativas da fitossociologia. O método de Prodan é tão eficiente quanto o de Área Fixa para estimativas de número de árvores, área basal e volume por hectare.

- PALAVRAS-CHAVE: Inventário florestal; unidade amostral; savana.

\section{Introdução}

O manejo de florestas naturais e a sua própria conservação demandam conhecimentos sobre sua estrutura e estoque corrente, bem como os fatores que determinam sua evolução ao longo dos anos (UBIAGLLI et al., 2009). E embora a medição de árvores demande muito tempo e recursos financeiros, esta é a base para obtenção de informações quantitativas e qualitativas da vegetação em determinado local

\footnotetext{
${ }^{1}$ Universidade de Brasília - UnB, Departamento de Engenharia Florestal, CEP: 70904-970, Brasília, DF, Brasil. E-mail: karolina.czs@gmail.com; hugo.efl@gmail.com; fabriciacm@yahoo.com.br; alba.rezende@gmail.com

${ }^{2}$ Universidade Federal Uberlândia - UFU, Instituto de Ciências Agrárias, Campus Monte Carmelo, CEP: 38500000, Monte Carmelo, MG, Brasil. E-mail: lidiomar.ef@gmail.com

${ }^{3}$ Instituto Federal de Brasília - IFB, Campus Planaltina, Caixa-Postal: 08202, CEP: 73380-900 - Brasília, DF, Brasil. E-mail: ilvanjunior@gmail.com

${ }^{4}$ Instituto Estadual de Florestas - IEF-MG, Escritório Regional Nordeste, CEP 39800-000, Teófilo Otoni, MG, Brasil. E-mail: murtaengflo@gmail.com
} 
(PÉLLICO NETTO e BRENA, 1997; RETSLAFF et al., 2014). Para tanto, o ideal é que a coleta de informações da vegetação seja precisa e de baixo custo (PEREIRA et al., 2015).

Para a obtenção dos parâmetros de uma floresta é necessária à medição de todos os indivíduos, o que é inviável na maioria das vezes (CAMPOS e LEITE, 2017; HUSCH et al., 2017), em virtude das limitações de recursos financeiros, tempo, mão-de-obra, acesso e tamanho das florestas (UBIAGLLI et al., 2009). Assim, realizar estimativas dos parâmetros, utilizando-se da amostragem da população, torna-se quase sempre, uma opção necessária (FARIAS et al., 2002). Tradicionalmente as técnicas de amostragem podem ser classificadas de duas diferentes formas: métodos de área fixa e métodos de área variável (SANQUETA et al., 2014).

No método de área fixa a seleção dos indivíduos é feita proporcional à área da unidade de amostra e, consequentemente, à frequência dos indivíduos que nela ocorrem (PÉLLICO NETTO e BRENA, 1997). Este método é o mais antigo, conhecido e utilizado em inventários florestais devido à simplicidade de utilização e pela gama de estimativas possíveis de uso segundo essa metodologia (SANQUETA et al., 2014). Entretanto, dentre suas desvantagens, tem-se alto custo de deslocamento para demarcação de parcelas, além dos riscos de erros na demarcação das mesmas.

Os métodos de área variável são preferíveis quando se deseja rapidez e eficiência, pois não há necessidade de demarcação de parcelas. Dentre esses, o método de Prodan (1968) ainda é pouco usado em inventários florestais no Brasil e fundamenta-se na inclusão de árvores na unidade amostral segundo probabilidade proporcional à distância da árvore ao centro da unidade amostral. Em termos práticos, esse método consiste e medir as cinco árvores mais próximas do ponto central e também a sexta que é considerada árvore marginal (SANQUETA et al., 2014).

Existem diversos estudos comprovando a eficácia de métodos alternativos de amostragem em florestas naturais, da Amazônia (OLIVEIRA et al., 2015; UBIALLI et al., 2009), Mata Atlântica (TÉO et al., 2014; NASCIMENTO et al., 2014; DIAS e COUTO, 2005); Mata de Araucária (MOSCOVICH et al., 1999), Cerradão (LIMA et al., 2017) e Mata de Galeria (BUENO et al., 2011). Entretanto, no Cerrado ainda não existem estudos suficientes, principalmente para o Cerrado sensu stricto, em que as pesquisas que objetivam a comparação entre métodos de amostragem alternativos ainda são escassas. É importante considerar o Cerrado como bioma prioritário para conservação, pois, possui alta riqueza de espécies e elevada heterogeneidade florística e fitofisionômica em escala espacial, local e regional (MENDONÇA et al., 2008; RIBEIRO e WALTER, 2008). Devido à grande área de distribuição original do Cerrado e à acelerada destruição que o bioma vem experimentando, é necessário conduzir estudos de campo que sejam rápidos e eficientes na descrição da composição e riqueza de espécies e da estrutura das comunidades de plantas nas áreas remanescentes.

Assim, o objetivo deste estudo foi avaliar e comparar a suficiência amostral, composição florística, estimadores de número de árvores, área basal e volume por hectare para os métodos de amostragem de Área Fixa e Prodan em uma área de Cerrado sensu stricto, no Distrito Federal. 


\section{Material e métodos}

A área de estudo está localizada no Distrito Federal, especificamente na Reserva Ecológica e Experimental da Universidade de Brasília, Fazenda Água Limpa (FAL) (Figura 1), situada a uma altitude de $1100 \mathrm{~m}$, entre as coordenadas $15^{\circ} 56^{\prime}$ e $15^{\circ} 59^{\prime} \mathrm{S}$ e $47^{\circ} 55^{\prime}$ e $47^{\circ} 58^{\prime} \mathrm{W}$.

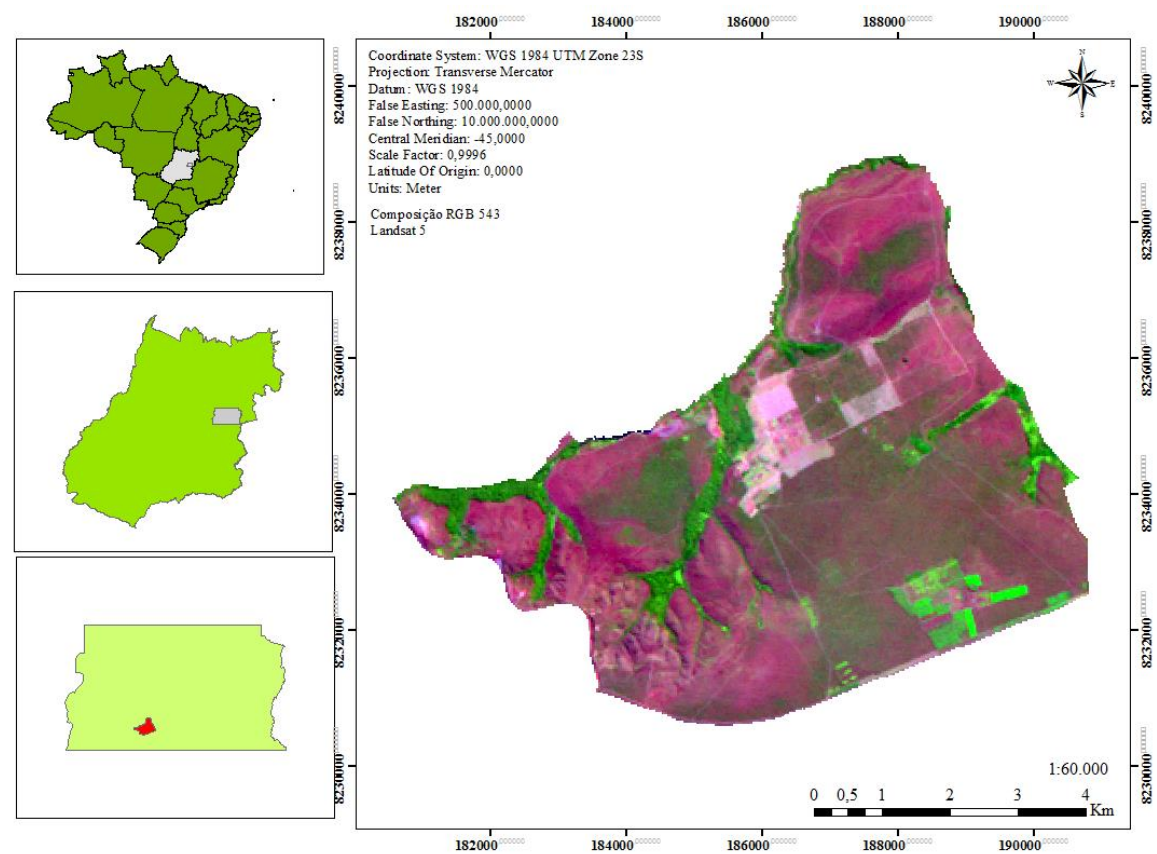

Figura 1 - Mapa da Fazenda Água Limpa onde se localiza a área amostrada no cerrado sensu stricto em Brasília, DF.

O clima da região é classificado em Aw de Köppen com temperaturas variando de $12^{\circ} \mathrm{C}$ a $28,5^{\circ} \mathrm{C}$. A umidade relativa entre maio e setembro fica abaixo de $70 \%$, ocorrendo mínima no mês de agosto, com uma média de $47 \%$, enquanto a precipitação média anual é de $1.600 \mathrm{~mm}$ (RAMOS et al., 2009).

A Fazenda Água Limpa possui uma área de aproximadamente 4000 ha e solos profundos, sendo predominante o Latossolo Vermelho Amarelo (EMBRAPA, 2013). A vegetação predominante é o Cerrado sensu stricto, que ocupa uma área de 1.480 ha, mas existem áreas cobertas por diversas fitofisionomias que variam desde campo limpo até matas de galeria.

Para coleta de dados, foram distribuídas aleatoriamente dez unidades amostrais na área de estudo para cada método de amostragem, sendo adotadas unidades amostrais retangulares com dimensões de $20 \times 50 \mathrm{~m}\left(1000 \mathrm{~m}^{2}\right)$ para o método de amostragem de Área Fixa e, para o método de Prodan, os pontos foram demarcados exatamente no centro de cada unidade amostral de área fixa. 
Em cada unidade amostral, para ambos os métodos, foram identificados e mensurados os indivíduos lenhosos vivos com diâmetro com casca, tomado a $0,30 \mathrm{~m}$ do nível do solo $(D b)$, igual ou superior a $5 \mathrm{~cm}$, além da altura total desses indivíduos. Os indivíduos mensurados foram classificados conforme o sistema Angiosperm Phylogeny Group IV (APG IV, 2016).

Para o cálculo do volume das árvores individuais, amostradas pelos dois métodos, utilizou-se a equação volumétrica desenvolvida por Rezende et al. (2006) para o Cerrado sensu stricto (Eq. 1).

$$
V=0,000109 \cdot D b^{2}+0,000451 \cdot\left(D b^{2} \cdot H t\right)\left(R^{2}=98,01 \% ; S_{y x}=25 \%\right)
$$

Para o método de Prodan, em cada ponto selecionou-se as seis árvores mais próximas e mediu-se a distância radial da sexta árvore, sendo esta distância utilizada para os cálculos dos estimadores das unidades amostrais.

A suficiência amostral foi analisada de acordo com a representação gráfica do número de espécies por número de unidades amostrais para os dois métodos de amostragem, conforme Mueller-Dombois e Ellemberg (1974) e Téo et al. (2014). No método de Área Fixa, conversão das estimativas amostrais para hectare foi feita utilizando o fator de proporcionalidade (Eq. 2), proposto por Péllico Netto e Brena (1997).

$$
F=\frac{A}{a}
$$

em que: $F$ é o fator de proporcionalidade; $A$ é a área de um hectare; e $a$ é a área da unidade amostral.

Para o cálculo da estimativa da área basal por hectare, do número de árvores por hectare e do volume por hectare no método de Área Fixa, seguiu-se a metodologia proposta por Moscovich et al. (1999), em que:

a) A estimativa da área basal por hectare $(G)$ foi obtida pelo somatório das áreas seccionais das $(n)$ árvores que ocorreram na unidade amostral multiplicado pelo fator de proporcionalidade $(F)($ Eq. 3):

$$
G=\left(\sum_{i=1}^{n} g_{i}\right) \cdot F
$$

b) O número de árvores por hectare $(N)$ foi obtido multiplicando-se o número de árvores medido na unidade amostral ( $n$ ) pelo fator de proporcionalidade (Eq. 4):

$$
N=n \cdot F
$$


c) $\mathrm{O}$ volume por hectare $(V)$ foi obtido pela soma dos volumes individuais $\left(v_{i}\right)$ referentes as $n$ árvores medidas na unidade e multiplicado pelo fator de proporcionalidade (Eq. 5).

$$
V=\left(\sum_{i=1}^{n} v_{i}\right) \cdot F
$$

Para o método de Prodan, a estimativa da área basal por hectare $(G)$, número de árvores por hectare $(N)$ e volume por hectare $(V)$ foi obtida através da metodologia encontrada em Péllico Netto e Brena (1997), em que:

a) A estimativa da área basal por hectare $(G)$ (Eq. 6):

$$
G=\frac{D b_{1}^{2}+D b_{2}^{2}+D b_{3}^{2}+D b_{4}^{2}+D b_{5}^{2}+\frac{D b_{6}^{2}}{2}}{R_{6}^{2}} \cdot 2500
$$

em que: $D b_{i}$ é diâmetro com casca tomado a $0,30 \mathrm{~m}$ do nível do solo de cada árvore da unidade amostral; e $R_{6}$ : raio da sexta árvore medida.

b) O número de árvores por hectare $(N)$ (Eq. 7):

$$
N=\frac{55000}{\pi \cdot R_{6}^{2}}
$$

c) A estimativa do volume por hectare $(V)$ foi obtida a partir do volume individual $\left(v_{i}\right)$ de cada uma das seis árvores amostradas, através da expressão (Eq. 8):

$$
V=\frac{v_{1}+v_{2}+v_{3}+v_{4}+v_{5}+\frac{v_{6}}{2}}{\pi \cdot R_{6}^{2}} \cdot 10000
$$

Para a comparação dos métodos de amostragem, foi considerado delineamento inteiramente casualizado, em que os métodos de amostragem constituíram os tratamentos, e os valores obtidos para cada unidade amostral, as repetições. Essa análise estatística foi realizada para o número de árvores por hectare, área basal por hectare e volume por hectare. Quando da ocorrência de diferenças significativas pelo teste de F a 5\% de significância entre esses valores estimados, efetuou-se o teste de comparação de médias de Tukey a 5\% de significância. Para realização da análise de variância, utilizou-se o software Statistica 12 (STATSOFT, 2013). 


\section{Resultados e discussão}

Para ambos os métodos de amostragem avaliados, os gráficos apresentam variações, porém sem tendência clara quanto à estabilização da relação entre o número de espécies observadas e o número de unidades amostrais. Esse fato sugere que, para o Cerrado sensu stricto, a medição de um número maior de unidades amostrais pode proporcionar uma relação número de espécie e unidade amostral com tendência de estabilização mais característica (Figura 2).

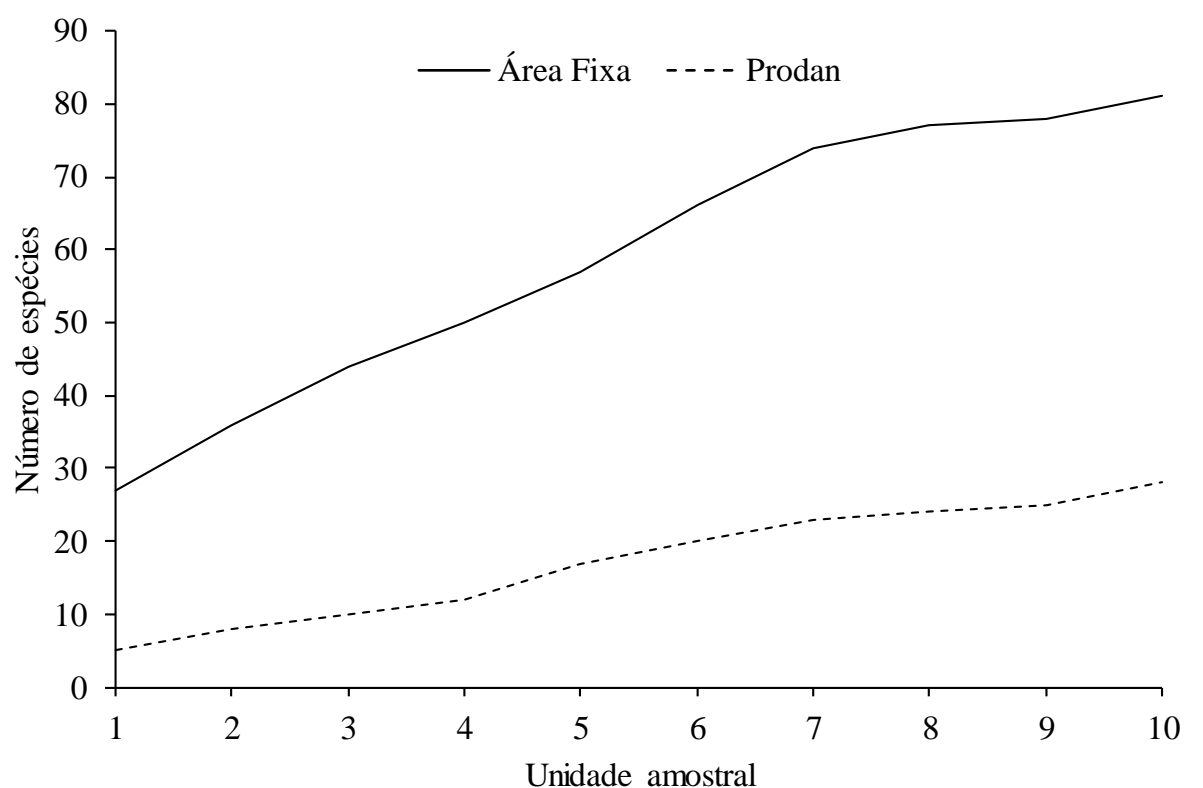

Figura 2 - Relação entre o número de espécies e unidades amostrais para os métodos de amostragem de Área Fixa e Prodan, em Cerrado sensu stricto, Brasília, DF.

No método de amostragem de Área Fixa observou-se tendência à estabilização do número de espécies amostradas entre a unidades amostrais 8 e 9 , porém observa-se uma retomada da tendência de aumento do número de espécies a partir $9^{\mathrm{a}}$ unidade amostral. Essa tendência sugere que a demarcação de poucas unidades amostrais adicionais seria suficiente para estabilizar a curva de suficiência amostral.

Para o método de Prodan, a curva de suficiência amostral apresenta pequeno crescimento do número de espécies com o aumento do número de unidades amostrais, porém, esse crescimento é constante e, por isso seria necessário maior número de unidades amostrais para estabilizar a curva. Estes resultados são coerentes com os encontrados em estudos em floresta ombrófila mista e floresta de Araucaria angustifolia comparando métodos de amostragem de área fixa e área variável (MOSCOVICH et al., 1999; TÉO et al., 2014). 
Utilizando o método de Área Fixa foram amostradas 69 espécies, já pelo método de Prodan foram amostradas 27 espécies (Tabela 1), ou seja, 39,1\% das espécies amostradas pelo método da área fixa também o foram pelo método de Prodan. Para o método de Área Fixa foram observadas espécies de 28 famílias botânicas e para o método de Prodan foram observadas espécies de 17 famílias. Lima et al. (2017), estudando métodos de amostragem de área fixa e variável em fragmento de cerradão no estado do Tocantins, observaram pelo método de Prodan 50,7\% das espécies e de $64,5 \%$ das famílias obtidas pelo método de área fixa. Deve-se levar em consideração que aqueles autores utilizaram 27 unidades amostrais para cada método. Dessa forma, para o método de área fixa a área amostrada é muito próxima àquela do presente trabalho, porém, quase três vezes maior quando se trata do método de Prodan. Por último, é também válido considerar que o cerradão é uma fitofisionomia com maior densidade de indivíduos arbóreos.

A Tabela 1 apresenta dados de frequência, densidade e dominância para parcelas de área fixa com o intuito de demonstrar que a maioria das espécies amostradas pelo método de Prodan são aquelas que possuem os maiores valores de frequência, densidade e dominância relativas calculadas no método de Área Fixa. Para dominância, por exemplo, das 25 espécies com maiores valores, 21 dessas foram amostradas também pelo método de Prodan. 
Tabela 1 - Família botânica e suas respectivas espécies observadas em cada um dos métodos de amostragem, além de frequência (FR), densidade (DR) e dominância (DoR) relativas das espécies observadas em parcelas de área fixa em cerrado sensu stricto no Distrito Federal

\begin{tabular}{|c|c|c|c|c|c|c|c|}
\hline \multirow{2}{*}{ Família } & \multirow{2}{*}{ Espécie } & \multicolumn{5}{|c|}{ Área Fixa } & \multirow{2}{*}{$\begin{array}{l}\text { Prodan } \\
\text { Ocorre }\end{array}$} \\
\hline & & FR & DR & DoR & IVI & Ocorre & \\
\hline Anonnaceae & Annona crassiflora & 0,32 & 0,07 & 0,11 & 0,50 & $\mathrm{x}$ & \\
\hline \multirow[t]{4}{*}{ Apocynaceae } & Aspidosperma macrocarpon & 1,27 & 0,41 & 0,26 & 1,94 & $\mathrm{x}$ & \\
\hline & Aspidosperma sp, & 0,63 & 0,34 & 0,23 & 1,20 & $\mathrm{x}$ & \\
\hline & Aspidosperma tomentosum & 1,27 & 0,54 & 0,34 & 2,15 & $\mathrm{x}$ & \\
\hline & Hancornia speciosa & 0,63 & 0,27 & 0,15 & 1,05 & $\mathrm{x}$ & \\
\hline Araliaceae & Schefflera macrocarpa & 3,16 & 2,99 & 4,17 & 10,32 & $\mathrm{x}$ & $\mathrm{x}$ \\
\hline \multirow[t]{2}{*}{ Asteraceae } & Eremanthus erythropappus & 0,32 & 0,14 & 0,1 & 0,56 & $\mathrm{x}$ & \\
\hline & Eremanthus glomerulatus & 1,9 & 0,61 & 0,33 & 2,84 & $\mathrm{x}$ & \\
\hline \multirow[t]{2}{*}{ Bignoniaceae } & Handroanthus ocraceus & 2,22 & 0,68 & 0,41 & 3,31 & $\mathrm{x}$ & \\
\hline & Tabebuia aurea & 0,32 & 0,07 & 0,03 & 0,42 & $\mathrm{x}$ & \\
\hline Caryocaraceae & Caryocar brasiliensis & 3,16 & 4,55 & 6,37 & 14,08 & $\mathrm{x}$ & $\mathrm{x}$ \\
\hline \multirow[t]{2}{*}{ Celastraceae } & Plenckia populnea & 0,32 & 0,07 & 0,09 & 0,48 & $\mathrm{x}$ & \\
\hline & Salacia crassifolia & 1,58 & 0,68 & 1,3 & 3,56 & $\mathrm{x}$ & \\
\hline \multirow[t]{3}{*}{ Clusiaceae } & Kielmeyera coriacea & 0,63 & 0,82 & 0,44 & 1,89 & $\mathrm{x}$ & \\
\hline & Kielmeyera lathrophyton & 0,32 & 0,41 & 0,21 & 0,94 & $\mathrm{x}$ & \\
\hline & Kielmeyera speciosa & 2,53 & 4,28 & 2,05 & 8,86 & $\mathrm{x}$ & $\mathrm{x}$ \\
\hline Combretaceae & Terminalia argentea & 0,32 & 0,07 & 0,03 & 0,42 & $\mathrm{x}$ & \\
\hline \multirow{2}{*}{ Connaraceae } & Connarus suberosum & 1,58 & 0,61 & 0,31 & 2,50 & $\mathrm{x}$ & \\
\hline & Rourea induta & 0,95 & 0,54 & 0,23 & 1,72 & $\mathrm{x}$ & \\
\hline Dilleniaceae & Davilla elliptica & 0,32 & 0,27 & 0,1 & 0,69 & $\mathrm{x}$ & $\mathrm{x}$ \\
\hline \multirow[t]{2}{*}{ Ebenaceae } & Diospyros burchellii & 0,32 & 0,27 & 0,14 & 0,73 & $\mathrm{x}$ & \\
\hline & Diospyros hispida & 1,27 & 0,27 & 0,17 & 1,71 & $\mathrm{x}$ & \\
\hline \multirow[t]{2}{*}{ Erythroxylaceae } & Erythroxylum deciduum & 0,32 & 0,07 & 0,02 & 0,41 & $\mathrm{x}$ & \\
\hline & Erythroxylum suberosum & 2,22 & 1,36 & 0,67 & 4,25 & $\mathrm{x}$ & \\
\hline \multirow[t]{2}{*}{ Fabaceae } & Bowdichia virgilioides & 1,27 & 0,68 & 0,48 & 2,43 & $\mathrm{x}$ & $\mathrm{x}$ \\
\hline & Dalbergia miscolobium & 3,16 & 4,89 & 6,19 & 14,24 & $\mathrm{x}$ & $\mathrm{x}$ \\
\hline \multirow[t]{13}{*}{ Fabaceae } & Dimorphandra mollis & 2,22 & 0,68 & 1,21 & 4,11 & $\mathrm{x}$ & $\mathrm{x}$ \\
\hline & Enterolobium gummiferum & 1,58 & 0,41 & 0,61 & 2,60 & $\mathrm{x}$ & \\
\hline & Hymenaea stigonocarpa & 0,63 & 0,27 & 0,28 & 1,18 & $\mathrm{x}$ & \\
\hline & Inga virescens & 0,32 & 0,07 & 0,13 & 0,52 & $\mathrm{x}$ & \\
\hline & Machaerium opacum & 0,32 & 0,48 & 0,31 & 1,11 & $\mathrm{x}$ & \\
\hline & Mimosa caesalpiniifolia & 0,32 & 0,07 & 0,02 & 0,41 & $\mathrm{x}$ & \\
\hline & Mimosa sp. & 0,32 & 0,07 & 0,05 & 0,44 & $\mathrm{x}$ & \\
\hline & Pterocarpus rotundifolius & 1,27 & 0,68 & 0,5 & 2,45 & $\mathrm{x}$ & $\mathrm{x}$ \\
\hline & Pterodon pubescens & 2,85 & 2,04 & 2,44 & 7,33 & $\mathrm{x}$ & $\mathrm{x}$ \\
\hline & Sclerolobium paniculatum & 2,22 & 1,63 & 4,58 & 8,43 & $\mathrm{x}$ & $\mathrm{x}$ \\
\hline & Stryphnodendron adstringens & 3,16 & 2,11 & 2,55 & 7,82 & $\mathrm{x}$ & $\mathrm{x}$ \\
\hline & Tachigali rugosa & 0,63 & 0,34 & 0,15 & 1,12 & $\mathrm{x}$ & \\
\hline & Vatairea macrocarpa & 0,32 & 0,27 & 0,51 & 1,10 & $\mathrm{x}$ & \\
\hline Loganiaceae & Strychnos pseudoquina & 0,63 & 0,2 & 0,33 & 1,16 & $\mathrm{x}$ & $\mathrm{x}$ \\
\hline
\end{tabular}


Continua...

Tabela 1 (continuação) - Família botânica e suas respectivas espécies observadas em cada um dos métodos de amostragem, além de frequência (FR), densidade (DR) e dominância (DoR) relativas das espécies observadas em parcelas de área fixa em cerrado sensu stricto no Distrito Federal

\begin{tabular}{|c|c|c|c|c|c|c|c|}
\hline \multirow[t]{5}{*}{ Malpighiaceae } & Byrsonima coccolobifolia & 1,58 & 1,09 & 0,56 & 3,23 & $\mathrm{x}$ & \multirow{5}{*}{$\mathrm{x}$} \\
\hline & Byrsonima pachyphylla & 2,53 & 2,86 & 2,04 & 7,43 & $\mathrm{x}$ & \\
\hline & Byrsonima verbascifolia & 1,9 & 0,54 & 0,39 & 2,83 & $\mathrm{x}$ & \\
\hline & Heteropterys byrsonimifolia & 0,63 & 0,2 & 0,1 & 0,93 & $\mathrm{x}$ & \\
\hline & Heteropterys sp. & 0,32 & 0,34 & 0,2 & 0,86 & $\mathrm{x}$ & \\
\hline Malvaceae & Eriotheca pubescens & 2,53 & 2,52 & 3,69 & 8,74 & $\mathrm{x}$ & $\mathrm{x}$ \\
\hline \multirow[t]{3}{*}{ Melastomataceae } & Miconia burchellii & 0,32 & 0,14 & 0,08 & 0,54 & $\mathrm{x}$ & \\
\hline & Miconia ferruginata & 2,22 & 1,97 & 1,45 & 5,64 & $\mathrm{x}$ & $\mathrm{x}$ \\
\hline & Miconia pohliana & 3,16 & 8,5 & 8,12 & 19,78 & $\mathrm{x}$ & $\mathrm{x}$ \\
\hline \multirow[t]{2}{*}{ Myrsinaceae } & Myrsine guianensis & 1,9 & 1,29 & 0,5 & 3,69 & $\mathrm{x}$ & \\
\hline & Rapanea guianensis & 1,27 & 2,58 & 1,12 & 4,97 & $\mathrm{x}$ & $\mathrm{x}$ \\
\hline \multirow[t]{2}{*}{ Myrtaceae } & Blepharocalyx salicifolius & 2,53 & 4,28 & 6,04 & 12,85 & $\mathrm{x}$ & $\mathrm{x}$ \\
\hline & Psidium laruotteanum & 0,32 & 0,07 & 0,05 & 0,44 & $\mathrm{x}$ & \\
\hline \multirow[t]{2}{*}{ Nyctaginaceae } & Neea theifera & 0,63 & 0,2 & 0,14 & 0,97 & $\mathrm{x}$ & \\
\hline & Guapira noxia & 1,27 & 0,41 & 0,26 & 1,94 & $\mathrm{x}$ & \\
\hline Ochnaceae & Ouratea hexasperma & 3,16 & 12 & 9,9 & 25,02 & $\mathrm{x}$ & $\mathrm{x}$ \\
\hline Proteaceae & Roupala montana & 3,16 & 2,92 & 2,01 & 8,09 & $\mathrm{x}$ & $\mathrm{x}$ \\
\hline Rubiaceae & Palicourea rigida & 2,22 & 1,16 & 0,7 & 4,08 & $\mathrm{x}$ & \\
\hline Sapotaceae & Pouteria ramiflora & 2,22 & 1,02 & 1,1 & 4,34 & $\mathrm{x}$ & $\mathrm{x}$ \\
\hline Styracaceae & Styrax ferrugineus & 2,53 & 1,77 & 1,77 & 6,07 & $\mathrm{x}$ & $\mathrm{x}$ \\
\hline Symplocaceae & Symplocos rhamnifolia & 0,32 & 0,07 & 0,03 & 0,42 & $\mathrm{x}$ & \\
\hline \multirow[t]{7}{*}{ Vochysiaceae } & Qualea grandiflora & 3,16 & 5,44 & 7,03 & 15,63 & $\mathrm{x}$ & $\mathrm{x}$ \\
\hline & Qualea multiflora & 2,85 & 1,29 & 1,05 & 5,19 & $\mathrm{x}$ & $\mathrm{x}$ \\
\hline & Qualea parviflora & 3,16 & 8,16 & 7,78 & 19,10 & $\mathrm{x}$ & $\mathrm{x}$ \\
\hline & Qualea sp. & 0,32 & 0,07 & 0,02 & 0,41 & $\mathrm{x}$ & \\
\hline & Vochysia elliptica & 2,53 & 1,84 & 1,07 & 5,44 & $\mathrm{x}$ & \\
\hline & Vochysia speciosa & 0,32 & 0,07 & 0,03 & 0,42 & $\mathrm{x}$ & \\
\hline & Vochysia thyrsoidea & 1,27 & 1,5 & 3,89 & 6,66 & $\mathrm{x}$ & \\
\hline
\end{tabular}

Moscovich et al. (1999) compararam os métodos de amostragem de Área Fixa, Bitterlich, Strand, Prodan e Quadrantes em floresta de Araucaria angustifolia e obtiveram uma abrangência de espécies maior para o método de Área Fixa. Os autores justificaram que os métodos de amostragem de Bitterlich, Strand, Prodan e Quadrantes amostraram as espécies mais representativas da fitossociologia da floresta e esse argumento também pode ser aplicado ao presente trabalho.

Para Dias e Couto (2005), a diferença entre o número de espécies amostradas pelos métodos deve-se principalmente ao número de indivíduos amostrados em cada método. Para os métodos de amostragem de área variável, dentre eles o método de Prodan, um 
menor número de árvores é amostrado em cada ponto, necessitando-se, portanto de maior intensidade amostral.

O número de árvores, área basal e volume por hectare não diferiram entre os métodos de amostragem (Tabela 2). Esse resulta corrobora com o observado por Téo et al. (2014) em fragmentos de Floresta Ombrófila Mista em Lebon Régis, SC. Aqueles autores, comparando métodos de amostragem de Área Fixa, Bitterlich, Strand e Prodan, observaram que as variáveis número de árvores por hectare e área basal por hectare não apresentaram diferenças significativas entre os métodos. O método de Prodan apresentou os maiores coeficientes de variação $(\mathrm{CV}, \%)$ entre unidades amostrais do que o método da Área Fixa para as três variáveis (Tabela 2). Isso demonstra que o número de árvores, a área basal e volume por hectare tiveram grandes oscilações entre unidades amostrais e que um maior número destas deve ser utilizado. Téo et al. (2014) relatam que esse resultado pode ser atribuído à forma de inclusão das árvores e pelo tamanho da unidade amostral de cada método.

Tabela 2 - Resultados obtidos pelos métodos de amostragem de Área Fixa e de Prodan para número de árvores por hectare $(\mathrm{N})$, área basal por hectare $\left(\mathrm{G}, \mathrm{m}^{2} \mathrm{ha}^{-1}\right) \mathrm{e}$ volume por hectare $\left(\mathrm{V}, \mathrm{m}^{3} \mathrm{ha}^{-1}\right)$, e seus respectivos coeficientes de variação $(\mathrm{CV}, \%)$ entre unidades amostrais em Cerrado sensu stricto em Brasília, DF

\begin{tabular}{ccc}
\hline \multirow{2}{*}{ Variável } & \multicolumn{2}{c}{ Método de Amostragem } \\
\cline { 2 - 3 } & Área Fixa & Prodan \\
\hline $\mathrm{N}^{\mathrm{ns}}$ & 1446,00 & 1758,41 \\
$\mathrm{CV}$ & 20,16 & 45,92 \\
$\mathrm{G}^{\mathrm{ns}}$ & 10,43 & 12,74 \\
$\mathrm{CV}$ & 13,25 & 48,80 \\
$\mathrm{~V}^{\mathrm{ns}}$ & 265,41 & 315,16 \\
$\mathrm{CV}$ & 18,50 & 60,12 \\
\hline$n s:$ diferença não significativa para o teste de F a 5\% de significância na análise de variância
\end{tabular}

Os valores do coeficiente de variação observados neste estudo são coerentes com os obtidos por Moscovich et al. (1999), em que para o método de Área Fixa, com 10 unidades amostrais, os valores de coeficientes de variação foram próximos de $10 \%$, enquanto que para o método de Prodan, com 25 unidades, observou-se valores em torno de 60\%. Já Miranda et al. (2015) observaram, pela abordagem de 30 unidades amostrais pelo método de Prodan, valores de coeficiente de variação entre 22,39 e 30,41\% para essas mesmas variáveis em plantio de teca, e isso contribui para que se possa atribuir essa diferença ao tipo de vegetação ou floresta e não propriamente ao método utilizado.

$\mathrm{Na}$ comparação de métodos de amostragem de área fixa e métodos outros métodos área variável, estudos disponíveis em literaturas pertinentes não relatam diferenças nas estimativas de área basal, volume e número de árvores por hectare. Dentre esses estudos têm-se diferentes fitofisionomias e/ou tipos de floresta, como por exemplo, Floresta Estacional Semidecidual, em Viçosa - MG (FARIAS et al., 2002), Floresta Ombrófila Densa (DIAS e COUTO, 2005), Floresta Ombrófila mista (Nascimento et al., 2015), 
plantio de Pinus taeda (DRUSZCZ et al., 2010) e plantios de Eucalyptus (SANTOS et al., 2013; SANTOS et al., 2016).

Uma vez observados e considerados os resultados obtidos por métodos de amostragem de área variável, especialmente o de Prodan, pode-se fazer as adaptações necessárias, como por exemplo, aumento do número de unidades amostrais ou adaptação do método para coleta de maior número de indivíduos por ponto amostrado. Além disso, considerando-se os custos dessas adaptações, esse método pode ser utilizado por apresentar maior eficiência com relação ao tempo estabelecimento de unidades amostrais e mensuração das plantas, além de amostrar as espécies mais representativas. Em se tratando do cerrado, pela sua vasta área de predominância e estado de antropização, estudos precisam ser conduzidos de forma rápida e eficiente para subsidiar medidas de conservação e manejo.

\section{Conclusões}

O método de Prodan, embora amostre menor número de espécies e famílias botânicas, contempla aquelas espécies mais representativas da fitossociologia, considerando os índices calculados pelo método de Área Fixa.

Os resultados obtidos pelo método de Prodan são compatíveis com aqueles obtidos utilizando o método de Área Fixa para as estimativas de número de árvores, área basal e volume por hectare.

\section{Agradecimentos}

Os autores agradecem os revisores e editores pelas sugestões e comentários que aprimoram o artigo.

OLIVEIRA, E. K. B.; COSTA, L. S.; LUSTOSA JÚNIOR, I. M.; MURTA JÚNIOR, L. S.; BUCHMANN, H. M.; MOTA, F. C. M.; REZENDE, A. V. Evaluation of Prodan method in the sampling of cerrado sensu stricto in the Distrito Federal. Rev. Bras. Biom., Lavras, v.37, n.4, p.467480, 2019.

- ABSTRACT: This study aimed to evaluate and compare the sampling methods of Prodan and Fixed Area (plots) in Cerrado sensu stricto in the Distrito Federal, Brazil. For the Fixed Area method, 10 plots of $20 \times 50 \mathrm{~m}$ were randomly distributed in the study area. In the center of each fixed area plot, points were marked for sampling by the Prodan method. In each sample unit, the trees were identified and the diameter at the base $(\mathrm{Db} \geq 5 \mathrm{~cm})$ and total height were measured. By Prodan method were sampled $39.1 \%$ of plant species and $60.7 \%$ of the botanical families that were sampled by the Fixed Area method. The plant species sampled by Prodan method were, mostly, those with higher relative values of frequency, density and dominance obtained in the Fixed Area method. The number of trees, basal area and volume per hectare did not differ between the two methods. Although the Prodan method obtain smaller samples in number of botanical species and families, it gets samples of the most representative species of phytosociology. The Prodan method is as efficient as the Fixed Area method for estimating tree numbers, basal area, and volume per hectare.

- KEYWORDS: Forest inventory; sampling unit; savanna. 


\section{Referências}

APG IV. An update of the Angiosperm Phylogeny Group classification for the orders and families of flowering plants: APG IV. Botanical Journal of the Linnean Society. v.181, n.1, p.1-20, 2016.

BUENO, B.; GALLETTI, V. S.; VICENTIN, W.; DAMASCENOJUNIOR, G. A.; POTT, A. Comparação de dois métodos fitossociológicos aplicados à mata ciliar do rio Miranda, Pantanal do Miranda, Corumbá, MS. In: FELFILI, J. M.; EISENLOHR, P. V.; MELO, M. M. R. F.; ANDRADE, L. A.; MEIRA-NETO, J. A. A. (Ed.). Fitossociologia no Brasil Métodos e estudo de caso. Viçosa: Universidade Federal de Viçosa, 2011. p. 86-121.

CAMPOS, J. C. C.; LEITE, H. G. Mensuração florestal: perguntas e respostas. Viçosa: UFV, 2017. 636p.

DIAS, A. C.; COUTO, H. T. Z. Comparação de métodos de amostragem na Floresta Ombrófila Densa - Parque Estadual Carlos Botelho/SP - Brasil. Revista do Instituto Florestal, v.17, n.1, p.63-72, 2005.

DRUSZCZ, J. P.; NAKAJIMA, N. Y.; PÉLLICO NETTO, S.; YOSHITANI JÚNIOR, M. Comparação entre os métodos de amostragem de Bitterlich e de área fixa com parcela circular em plantação de Pinus taeda. Floresta, v.40, n.4, p.739-754, 2010.

EMPRESA BRASILEIRA DE PESQUISA AGROPECUÁRIA - EMBRAPA. Centro Nacional de Pesquisa do Solo. Sistema brasileiro de classificação de solos. Rio de Janeiro: Embrapa Solo, 2013. 353p.

FARIAS, C. A.; SOARES, C. P. B.; SOUZA A. L.; LEITE, H. G. Comparação de métodos de amostragem para análise estrutural de florestas inequiâneas. Revista Árvore, v.26, n.5, p.541-548, 2002.

GOMES, K. B. P.; MACHADO FILHO, V. A.; SILVA, V. P.; SILVA, A. G. Comparação dos métodos de amostragem casual simples: Bitterlich $\mathrm{e}$ Prodan. Revista Agrogeoambiental, v.3, n.1, p.101-104, 2011.

HUSCH, B.; BEERS, T. W.; DUCEY, M. J.; KERSHAW JR, J. A. Forest mensuration. New Jersey: John Wiley \& Sons, 5 ed. 2017, 632 p.

LIMA, M. B.; MIGUEL, E. P.; MEIRA JÚNIOR, M.S.; NAPPO, M.E.; REZENDE, A. V.; MATIAS, R.A. Comparison of sampling methods for description of floristic-structure in woody vegetation. Australian Journal of Crop Science. v.11, n.12, p. 1573-1578, 2017.

MENDONÇA, R. C.; FELFILI, J. M.; WALTER, B. M. T.; SILVA JR, M. C.; REZENDE, A. B.; FILGUEIRAS, T. S.; NOGUEIRA, P. E.; FAGG, C. W. Flora vascular do Bioma Cerrado: checklist com 12.356 espécies. In: SANO, S. M.; ALMEIDA, S. P.; RIBEIRO, J. F. (Org.). Cerrado: Ecologia e Flora. Brasília, DF: Embrapa, 2008. p.213228.

MIRANDA, D. L. C.; FRANCIO, J.; SANTOS, J. P.; SANQUETTA, C. R.; DALlA CORTE, A. P. Precisão e eficiência relativa de métodos de amostragem em teca. Pesquisa Florestal Brasileira, v.35, n.83, p.247-254, 2015. 
MOSCOVICH, F. A.; BRENA, D. A.; LONGHI, S. J. Comparação de diferentes métodos de amostragem, de área fixa e variável, em uma floresta de Araucaria angustifolia. Ciência Florestal, v.9, n.1, p.173-191, 1999.

MUELLER-DOMBOIS, D.; ELLENBERG, H. Aims and methods of vegetation ecology. New York: John Wiley \& Sons, 1974. 547p.

NASCIMENTO, R. G. M.; SILVA, L. C. R.; BARBEIRO, L. S. S.; WOJCIECHOWSKI, J. C.; PÉLLICO NETTO, S.; MACHADO, S. A. Efeito da árvore marginal nos estimadores populacionais obtidos por métodos de amostragem de área variável. Cerne, v.21, n.1, p.125-131, 2015.

OLIVEIRA, G. S.; CAVALCANTI, F. J. B.; MELO, L. A.; MONTE, M. A. O Método de quadrantes para estimativa de variáveis quantitativas em Planos de Manejo na Amazônia. Cerne, v. 21, n.4, p. 683-692, 2015.

PÉLLICO NETTO, S.; BRENA, D. A. Inventário florestal. Curitiba: Universidade Federal do Paraná - Universidade Federal de Santa Maria, 1997. 316p.

PEREIRA, F. C.; LENZA, E.; MARACAHIPES-SANTOS, L.; MEWS, H. A.; GOMES, L.; LIMA, S. L.; SILVA MELO SANTOS, K. S. M. Comparação dos métodos de parcelas e pontos-quadrantes para descrever uma comunidade lenhosa de Cerrado Típico. Biotemas, v.28, n.2, p.61-72, 2015.

PRODAN, M. Forest biometrics. Oxford: Pergamon press, 1968. 447p.

RAMOS, A. M.; SANTOS, L. A. R.; FORTES, L. T. G. Normais Climatológicas do Brasil 1961-1990. Brasília, DF: Instituto Nacional de Meteorologia - INMET, Ministério da Agricultura, Pecuária e Abastecimento - MAPA, 2009. 465p.

RETSLAFF, F. A. S.; LANA, M. D.; DOUBRAWA, B.; FIGUEIREDO FILHO, A.; PÉLLICO NETTO, S. Amostragem em conglomerados pelo método de Bitterlich em floresta ombrófila mista. Nativa, v.2, n.4, p.393-402, 2014.

REZENDE, A. V.; VALE, A. T.; SANQUETTA C. R.; FIGUEIREDO FILHO, A.; FELFILI, J. M. Comparação de modelos matemáticos para estimativa do volume, biomassa e estoque de carbono da vegetação lenhosa de um cerrado sensu stricto em Brasília, DF. Scientia Forestalis, v.71, p.65-76, 2006.

RIBEIRO, J. F.; WALTER, B. M. T. As principais fitofisionomias do Bioma Cerrado. In: SANO, S. M.; ALMEIDA, S. P.; RIBEIRO, J. F. (Org.). Cerrado: ecologia e flora. Brasília, DF: Embrapa, 2008. p.151-212.

SANQUETA, C. R.; CORTE, A. P. D.; RODRIGUES, A. L.; WATZLAWICK, L. F. Inventários Florestais: Planejamento e Execução. Curitiba, PR: Multi-Graphic Gráfica e Editora, 2014. 406p.

SANTOS, F. E. V.; ARAÚJO, J. M.; ANDRADE, W. C. Comparação dos métodos de amostragem parcela de área fixa e Strand em floresta de eucalipto. Revista Verde de Agroecologia e Desenvolvimento Sustentável, v.8, n.1, p.174-177, 2013.

SANTOS, J. S.; MENDONÇA, A. R.; SILVA, G. F.; FRAGA FILHO, C. V. Método de amostragem de Bitterlich: uma alternativa de inventário florestal para pequenas propriedades rurais fomentadas. Revista Brasileira de Ciências Agrárias, v.11, n.1, p.46$52,2016$. 
STATSOFT, INC. Statistica (Data Analysis Software System), Version 12. www.statsoft.com. 2013.

TÉO, S. J.; SCHNEIDER, C. R.; FIORENTIN, L. D.; COSTA, R. H. Comparação de métodos de amostragem em fragmentos de floresta ombrófila mista, em Lebon Régis, SC. Floresta, v.44, n.3, p.393-402, 2014.

UBIALLI, J.A.; FIGUEIREDO FILHO, A.; MACHADO, S. A.; ARCE, J. E. Comparação de métodos e processos de amostragem para estudos fitossociológicos em uma floresta ecotonal na região norte mato-grossense. Floresta, v.39, n.3, p.511-523, 2009.

Recebido em 17.01.2019

Aprovado após revisão em 06.08.2019 